Nazila Esmaeili ${ }^{*}$, Alfredo Illanes, Axel Boese, Nikolaos Davaris, Christoph Arens, Nassir Navab and Michael Friebe

\title{
Manual versus Automatic Classification of Laryngeal Lesions based on Vascular Patterns in CE+NBI Images
}

\begin{abstract}
Longitudinal and perpendicular changes in the blood vessels of the vocal fold have been related to the advancement from benign to malignant laryngeal cancer stages. The combination of Contact Endoscopy (CE) and Narrow Band Imaging (NBI) provides intraoperative realtime visualization of vascular pattern in Larynx. The evaluation of these vascular patterns in $\mathrm{CE}+\mathrm{NBI}$ images is a subjective process leading to differentiation difficulty and subjectivity between benign and malignant lesions. The main objective of this work is to compare multi-observer classification versus automatic classification of laryngeal lesions. Six clinicians visually classified $\mathrm{CE}+\mathrm{NBI}$ images into benign and malignant lesions. For the automatic classification of $\mathrm{CE}+\mathrm{NBI}$ images, we used an algorithm based on characterizing the level of the vessel's disorder. The results of the manual classification showed that there is no objective interpretation, leading to difficulties to visually distinguish between benign and malignant lesions. The results of the automatic classification of $\mathrm{CE}+\mathrm{NBI}$ images on the other hand showed the capability of the algorithm to solve these issues. Based on the observed results we believe that, the automatic approach could be a valuable tool to assist clinicians to classifying laryngeal lesions.
\end{abstract}

Keywords: Contact Endoscopy, Larynx, Classification

*Corresponding author: Nazila Esmaeili: Institute of Medical Technology, Otto-von-Guericke University Magdeburg, Universitätspl. 2, Magdeburg, Germany, e-mail: nazila.esmaeili@ovgu.de

Alfredo Illanes, Axel Boese: Institute of Medical Technology, Otto-von-Guericke University Magdeburg, Magdeburg, Germany

Nikolaos Davaris, Christoph Arens: Department of Otorhinolaryngology, Head and Neck Surgery, Magdeburg University Hospital, Magdeburg, Germany

Nassir Navab: Institut für Informatik, Technische Universität München, München, Germany

Michael Friebe: Faculty of Medicine, Otto-von-Guericke University Magdeburg, Magdeburg, Germany, 5IDTM GmbH, Recklinghausen, Germany https://doi.org/10.1515/cdbme-2020-3018

\section{Introduction}

Laryngeal cancer is one of the most common tumors of the respiratory tract with a high incidence and mortality. Laryngeal lesions in a precancerous stage such as laryngeal dysplasia could develop to cancerous stage. Hence, early detection and diagnosis of suspicious laryngeal mucosal lesions is important to preserve the larynx and vocal fold function $[1,2]$.

Changes in the organization and structure of the vocal fold's blood vessels are directly related to the development of benign and subsequent malignant lesions. The Endoscopic Laryngeal Imaging section of the European Laryngological Society proposed a guideline for the classification of these changes and divided them into two main categories, longitudinal and perpendicular vascular changes [3]. Longitudinal vascular changes (LVC) spread along the length and width of the vocal fold and can be observed in all kinds of benign or malignant lesions. On the contrary, perpendicular vascular changes (PVC) develop perpendicularly towards the mucosa intraepithelial and are associated to papillomatosis and malignant lesions. The evaluation of LVC and PVC therefore has a high diagnostic relevance $[3,4]$.

Larynx endoscopy techniques can provide valuable information about the structural changes of the vocal fold's vessels in real time. Contact Endoscopy (CE) is one of these techniques that can be combined with other optical enhancement techniques such as Narrow Band Imaging (NBI) to provide a clear visualization of the vascular patterns of the larynx [5]. The first application of CE in the larynx was reported in 1995 and its efficiency was subsequently confirmed as a diagnostic tool in the evaluation of various pathologies in the larynx [6,7]. However, the evaluation of vascular patterns in $\mathrm{CE}+\mathrm{NBI}$ images depends on the clinicians' experience as at the beginning of the training there is a risk of subjective evaluation causing difficulty in differentiation between benign and malignant lesions [8-10]. 
The main objective of this work is to present a preliminary comparison of multi-observer manual classification versus automatic classification of $\mathrm{CE}+\mathrm{NBI}$ images into benign and malignant lesions. For that, a dataset of $\mathrm{CE}+\mathrm{NBI}$ was generated. The classification scenario presented in [11] was used for the manual classification including three experienced and three less-experienced Otolaryngologist who visually evaluated $\mathrm{CE}+\mathrm{NBI}$ images and subsequently classified them based on PVCs appearance in the images. For the automatic classification, we used the algorithm that was already proposed in our previous work [12] for automatic characterization of laryngeal lesions based on vascular patterns in $\mathrm{CE}+\mathrm{NBI}$ images.

\section{Material and Methods}

\subsection{Data Acquisition and Dataset Generation}

Video scenes of 68 patients with histologically examined laryngeal lesions were acquired. An Evis Exera III Video System with a xenon light source plus integrated NBI-filter (Olympus Medical Systems, Hamburg, Germany) and a rigid 30-degree contact endoscope (Karl Storz, Tuttlingen, Germany) were used. 1632 high resolution CE+NBI images with unique vascular patterns were manually extracted from these videos. The images were labelled into benign and malignant groups based on histological diagnosis according to the WHO classification [13].

Table 1 shows the histopathologies with their number of patients and images used for the generation of the dataset.

\subsection{Manual Classification}

Based on [11], a series of four to five CE+NBI images were randomly selected for each patient from the previously extracted images. Three otolaryngology specialists (experienced observers) and three otolaryngology residents (less- experienced observers), blinded to the histologic diagnosis, independently visually evaluated the series of images to detect and classify lesions based on vascular patterns. PVC-positive lesions with the malignant histological diagnosis were considered true positives for the calculation of sensitivity and specificity. The calculation was conducted with the IBM SPSS Statistics software package $(\mathrm{V}-26)$, and the average value for each group of observers was presented.
Table 1: Histopathologies used for the generation of the dataset.

\begin{tabular}{|c|c|c|}
\hline Type of Lesion & Histopathology & $\begin{array}{c}\text { Number of Patients I } \\
\text { Images }\end{array}$ \\
\hline \multirow{7}{*}{ Benign } & Cyst & $3 / 90$ \\
\hline & Polyp & $5 / 71$ \\
\hline & Reinke's edema & $12 / 329$ \\
\hline & Hyperkeratosis & $4 / 82$ \\
\hline & $\begin{array}{l}\text { Squamous } \\
\text { hyperplasia }\end{array}$ & $3 / 75$ \\
\hline & Papillomatosis & $11 / 286$ \\
\hline & Other lesions & $5 / 88$ \\
\hline \multirow{5}{*}{ Malignant } & Dysplasia mild & $3 / 77$ \\
\hline & $\begin{array}{l}\text { Dysplasia } \\
\text { moderate }\end{array}$ & $2 / 49$ \\
\hline & Dysplasia severe & $3 / 68$ \\
\hline & Carcinoma in situ & $9 / 249$ \\
\hline & $\begin{array}{c}\text { Squamous Cell } \\
\text { Carcinoma (SCC) }\end{array}$ & $8 / 168$ \\
\hline
\end{tabular}

\subsection{Automatic Classification}

An algorithm [12] was used for the automatic classification of CE+NBI images by characterizing the level of the vessel's disorder based on the consistency of gradient direction and the vessels' curvature.

For the image pre-processing and vessel segmentation step, the Jerman filter [14] was used as a new enhancement technique to overcome the observed deficiencies of the already used Frangi filter. Sigma $(\sigma)$ and Tau $(\tau)$ are two main parameters of the enhancement function in Jerman filter. $\sigma$ is a vector of scales on which the vesselness is computed. This parameter was set in the range of $0.5 \mathrm{~mm}$ to $2.5 \mathrm{~mm}$ with the step size of $0.5 \mathrm{~mm}$ to cover all the possible vascular structures. $\tau$ is a parameter that controls response uniformity. The empirical tests showed that a value between 0.5 to 1 is the proper value for $\tau$. For that, in this study $\tau$ was set as 1 . Figure 1 shows the outcome of this filter followed by the skeletonization process.

Five indicators were computed after image preprocessing and vessel segmentation based on direction-based and curvature-based characteristics of the vessels in CE+NBI images. Then 24 features were extracted based on the qualitative properties of these indicators as described in [12]. 
Features were fed into four supervised classifiers, including Support Vector Machine (SVM) with Polykernel and Radial Basis Function (RBF), k-Nearest Neighbor $(\mathrm{kNN})$, and Random Forest (RF). The optimal hyperparameters for each classifier were set based on the results of hyperparameter tuning in [12]. For the classification scenarios, the same CE+NBI images selected for the manual classification were used as the testing set (336 images, $\sim 20 \%$ of the dataset), and the rest of the images were used for training (1296 image, $\sim 80 \%$ of the dataset). We used this training and testing set strategy to be able to compare the results of manual and automatic classifications. The sensitivity and specificity were calculated from a confusion matrix for each classification scenario.
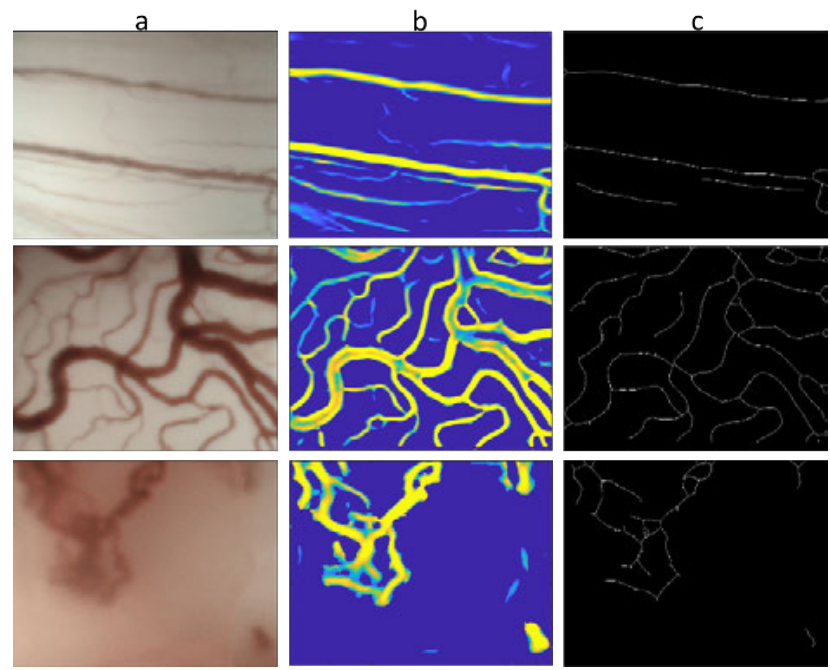

Figure 1: Imgae pre-processing: a) Original image, b) Jermann Filter, c) Skeletonization

\section{Results and Discussion}

Table 2 presents the results of manual classification performed by the clinical observers and automatic classification using four supervised classifiers.

The mean sensitivity value of manual classification showed a difference between experienced and lessexperienced groups. Showing that the interpretation of vascular patterns in $\mathrm{CE}+\mathrm{NBI}$ images is subjective and highly depends on the experiences of the clinicians. The mean specificity of 0.630 and 0.609 values in manual classification can be explained by the difficulty to visually distinguish between benign and malignant lesions based on vascular pattern. Papillomatosis is a benign histopathology that has similar vascular patterns to malignant lesions leading to misclassification of papillomatosis as malignant lesions. The automatic classification showed a sensitivity of 0.959 by SVM with RBF and specificity of 0.932 by $\mathrm{kNN}$. These results emphasize the ability of the automatic approach to solve the problem of misclassification of laryngeal lesions such as papillomatosis and with that is valuable for all clinicians, experienced and less- experienced ones.

Table 2: Results of manual classification versus automatic classification of $\mathrm{CE}+\mathrm{NBI}$ images.

\begin{tabular}{cccc}
\hline \multirow{4}{*}{$\begin{array}{c}\text { Manual } \\
\text { Classification }\end{array}$} & $\begin{array}{c}\text { Experienced } \\
\text { Observers }\end{array}$ & 0.955 & 0.727 \\
\cline { 2 - 4 } & $\begin{array}{c}\text { Less- } \\
\text { experienced } \\
\text { Observers }\end{array}$ & 0.630 & 0.609 \\
\hline & $\begin{array}{c}\text { SVM with } \\
\text { Polykernel }\end{array}$ & 0.819 & 0.872 \\
\cline { 2 - 4 } Automatic & $\begin{array}{c}\text { SVM with } \\
\text { RBF }\end{array}$ & 0.959 & 0.853 \\
\cline { 2 - 4 } Classification & kNN & 0.942 & 0.873 \\
\cline { 2 - 4 } & RF & 0.872 & 0.932 \\
\hline
\end{tabular}

\section{Conclusion}

The results confirm the relevance of the vascular patterns as an evaluation parameter for benign or malignant lesions. The automatic approach has the potential to operate as assisting system to provide a confident way for clinicians to make the final decision about the stage of the laryngeal cancer in the routine surgical procedures.

\section{Author Statement}

Research funding: The author state no funding involved. Conflict of interest: Authors state no conflict of interest. Informed consent: Informed consent has been obtained from all individuals included in this study. Ethical approval: The research related to human use complies with all the relevant national regulations, institutional policies and was performed in accordance with the tenets of the Helsinki Declaration, and has been approved by the authors' institutional review board or equivalent committee. 


\section{References}

[1] Steuer CE, El-Deiry M, Parks JR, Higgins KA, Saba NF. An update on larynx cancer. CA: a cancer journal for clinicians. 2017; 67(1):31-50.

[2] Holliday EB, Smith BD, Gross ND, Fuller CD, Rosenthal DI. Larynx Cancer. The American Cancer Society's Oncology in Practice: Clinical Management. 2018; 9:201.

[3] Arens C, Piazza C, Andrea M, Dikkers FG, Gi RE, VoigtZimmermann S, Peretti G. Proposal for a descriptive guideline of vascular changes in lesions of the vocal folds by the committee on endoscopic laryngeal imaging of the European Laryngological Society. European Archives of OtoRhino-Laryngology. 2016; 273(5):1207-14.

[4] Šifrer R, Rijken JA, Leemans CR, Eerenstein SE, van Weert S, Hendrickx JJ, Bloemena E, Heuveling DA, Rinkel RN. Evaluation of vascular features of vocal cords proposed by the European Laryngological Society. European Archives of Oto-Rhino-Laryngology.2018; 275(1):147-51.

[5] Puxeddu R, Sionis S, Gerosa C, Carta F. Enhanced contact endoscopy for the detection of neoangiogenesis in tumors of the larynx and hypopharynx. The Laryngoscope. 2015; 125(7):1600-6.

[6] Andrea M, Dias O, Santos A. Contact endoscopy during microlaryngeal surgery: a new technique for endoscopic examination of the larynx. Annals of Otology, Rhinology \& Laryngology. 1995; 104(5):333-9.

[7] Arens C, Voigt-Zimmermann S. Kontaktendoskopie der Stimmlippen in Kombination mit Narrow-Band-Imaging (Kompaktendoskopie). Laryngo-Rhino-Otologie. 2015; 94(03):150-2.
[8] Carta F, Sionis S, Cocco D, Gerosa C, Ferreli C, Puxeddu R. Enhanced contact endoscopy for the assessment of the neoangiogenetic changes in precancerous and cancerous lesions of the oral cavity and oropharynx. European Archives of Oto-Rhino-Laryngology. 2016;273(7):1895-903.

[9] Mannelli G, Cecconi L, Gallo O. Laryngeal preneoplastic lesions and cancer: challenging diagnosis. Qualitative literature review and meta-analysis. Critical reviews in oncology/hematology. 2016; 106:64-90.

[10] Piazza C, Cocco D, Del Bon F, Mangili S, Nicolai P, Peretti G. Narrow band imaging and high definition television in the endoscopic evaluation of upper aero-digestive tract cancer. Acta otorhinolaryngologica italica. 2011; 31(2):70.

[11] Davaris N, Lux A, Esmaeili N, Illanes A, Boese A, Friebe M, Arens C. Evaluation of Vascular Patterns Using Contact Endoscopy and Narrow-Band Imaging (CE-NBI) for the Diagnosis of Vocal Fold Malignancy. Cancers. 2020; 12(1):248.

[12] Esmaeili N, Illanes A, Boese A, Davaris N, Arens C, Friebe M. Novel automated vessel pattern characterization of larynx contact endoscopic video images. International journal of computer assisted radiology and surgery. 2019; 14(10):175161.

[13] Gale N, Hille J, Jordan RC, Nadal A, Williams MD. Regarding Laryngeal precursor lesions: Interrater and intrarater reliability of histopathological assessment. The Laryngoscope. 2019; 129(3):E91-2.

[14] Jerman T, Pernuš F, Likar B, Špiclin Ž. Enhancement of vascular structures in $3 \mathrm{D}$ and $2 \mathrm{D}$ angiographic images. IEEE transactions on medical imaging. 2016; 35(9):2107-18. 